\title{
A Vortex in an Egg Cell
}

\author{
During a fruit-fly egg cell's early development, its internal fluid begins to \\ swirl in a vortex-a transition caused by the coordinated behavior of \\ elastic filaments in the cell. \\ By Rachel Berkowitz
}

A $t$ the end of its first week of development, a striking change occurs in a fruit-fly egg cell. The cell's internal fluid motion transitions from a disordered mix of small-scale flows to a single vortex that encompasses the entire cell. Biologists have long recognized that cellular fluid is stirred by molecular motor proteins marching along a network of cell-spanning microtubules, but the cause of the vortex flow transition has remained unclear. Through fluid dynamics calculations, David Stein of the Flatiron Institute, New York, and colleagues now determine that microtubules self-organize in a way that drives a rotational flow field in the cellular fluid [1]. The findings provide a first quantitative description of an observed developmental transition in the fruit fly cell.

Stein and colleagues began with recent observations that-within the tangled microtubule network-some microtubules are attached to the cell boundary in an ordered arrangement. They developed a continuum model that treats the dense array of microtubules as a deformable porous medium and showed how molecular motors translating along microtubules lead to inward-pointing forces on the fluid in the

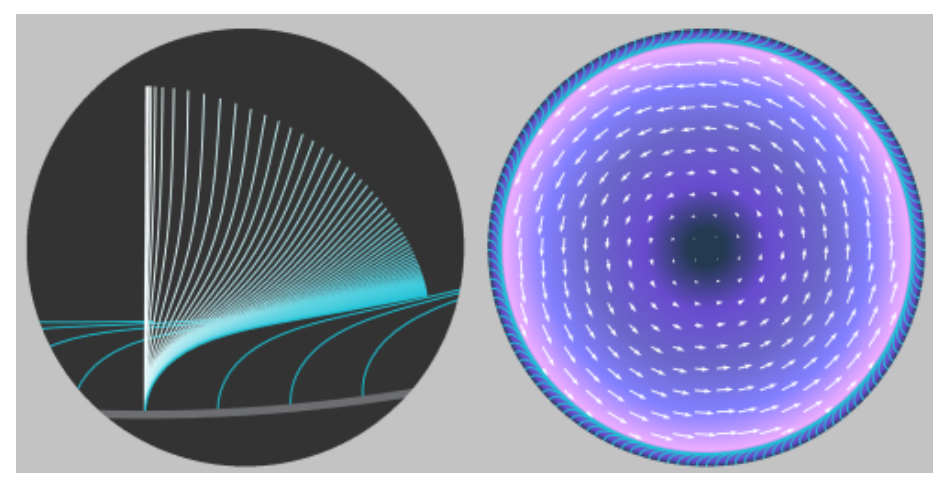

Credit: Lucy Reading-Ikkanda cell. Given the fluid's incompressibility, those forces can give rise to what the researchers call a "swirling" instability, and the flow switches to a cell-spanning rotation. The threshold of molecular motor activity for the transition depends on the buckling of individual microtubules, which are treated as elastic rods. By pinpointing the role of microtubule self-organization, the research opens the door to genetic studies that could explore how flow transitions affect the development of fruit flies, as well as other organisms that exhibit similar cellular behavior.

Rachel Berkowitz is a Corresponding Editor for Physics based in Vancouver, Canada.

\section{REFERENCES}

1. D. B. Stein et al., "Swirling instability of the microtubule cytoskeleton," Phys. Rev. Lett. 126, 028103 (2021). 